\title{
Modeling of Errors Realized by a Human Learner in Virtual Environment for Training
}

\author{
Thanh-Hai Trinh, Cédric Buche, Ronan Querrec, Jacques Tisseau
}

Université Européenne de Bretagne, Ecole Nationale d'Ingénieurs de Brest, Laboratoire Informatique des Systèmes Complexes, Centre Européen de Réalite Virtuelle Technopôle Brest-Iroise, 29238 Brest Cedex 3, France

E-mail: $\{$ trinh,buche,querrec,tisseau $\}$ enib.fr

\begin{abstract}
This study focuses on the notion of erroneous actions realized by human learners in Virtual Environments for Training. Our principal objective is to develop an Intelligent Tutoring System (ITS) suggesting pedagogical assistances to the human teacher. For that, the ITS must obviously detect and classify erroneous actions produced by learners during the realization of procedural and collaborative work. Further, in order to better support human teacher and facilitate his comprehension, it is necessary to show the teacher why learner made an error. Addressing this issue, we firstly modeling the Cognitive Reliability and Error Analysis Method (CREAM). Then, we integrate the retrospective analysis mechanism of CREAM into our existing ITS, thus enable the system to indicate the path of probable cause-effect explaining reasons why errors have occurred.

Keywords: Intelligent tutoring system, Erroneous actions, Retrospective analysis
\end{abstract}

\section{Introduction}

In order to simulate procedural and collaborative work, we developed the model MASCARET (Multi-Agent System for Collaborative Adaptive and Realistic Environment for Training) where human learners and agents collaborate to realize a task [1]. Learners are gathered in team consisting of several predefined roles, every role contains a number of actions to be realized by learners with specific resources. During realization of the tasks, it is essential to take into account that human learners may make erroneous actions in comparing to their predefined correct procedure.

In [2], we have proposed a model of Intelligent Tutoring System (ITS) whose principal objective is to suggest pedagogical assistances to the teacher adapted to the simulation context and to the learner's behaviours (including erroneous actions). However, this works exclusively concerns errors detection and tagging. Once erroneous actions are detected in our existing ITS, it were be classified in different types (cf. Figure 1(a)) whose explications are based on a knowledge base on classical errors. In order to better support the teacher and facilitate his comprehension, it lacks a model that could explain reasons why the learner made an error.

Our approach bases on the Cognitive Reliability and Error Analysis Method (CREAM) in Human

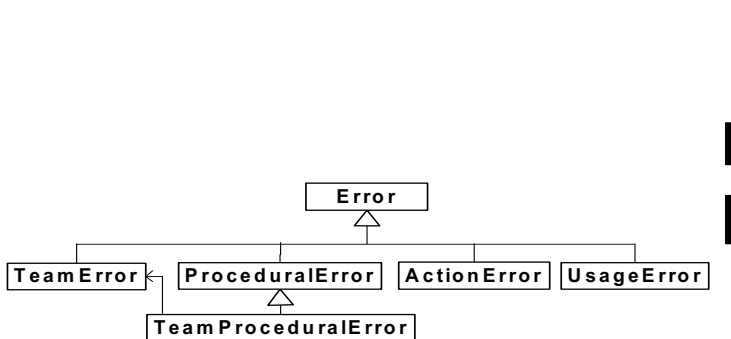

(a) Errors types in ITS [2]

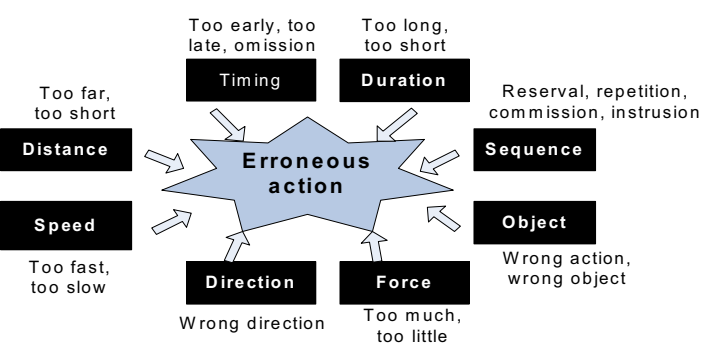

(b) Dimensions of error modes [3]

Figure 1: Errors types and errors's phenotypes

Reliability Analysis field [3]. This approach proposed a classification scheme which makes a distinction between observations of errors (phenotypes, cf. Figure 1(b)) and its causes (genotypes) classified in 
three categories: $\mathrm{M}(\mathrm{an}), \mathrm{T}($ echnology) and $\mathrm{O}$ (rganization). For example, since the learner made a mistake about the order of actions, the erroneous action observed is in phenotype Sequence and that can be futher explained by some genotypes such as Inattention (Man related genotype), Communication failure (Technology related genotype), etc. The causal links between phenotype-genotype are represented using a number of consequent-antecedent links. Finally, the scheme could be associated with both a method of retrospective analysis (the search for causes) and a performance prediction method. However, in our goal of erroneous actions detection and then searching for the causes, we interested in human learner's performance analyses, in other words, in retrospective analyses.

Implementation of CREAM was object in the work of El-Kechaï [4][5] which firstly proposed a task model named METISSE in order to recognize learner's plans in Virtual Environments for Training (VET), then this model could be used to detect for erroneous actions according to classification of Hollnagel. Nevertheless, implementation of METISSE was not complete, and integration of CREAM into a really ITS was not performed.

In this paper, we will firstly propose an approach to model CREAM (Section 2). Next, in Section 3, we will present the integration of retrospective analysis mechanism of CREAM into our existing ITS as well as our evaluation.

\section{Implementation of CREAM}

\subsection{Classification Scheme Representation}

There are several graphic tools that permit to keep track of analyses processes such as CREAM Navigator developed by Serwy and Rantanen [7]. However, this navigator is completely closed in the sense that it does not maintain an explicit representation of possible errors modes and probable causes. For that, [4] proposed using a rules base for represent consequent-antecedent links, hence the search for the causes was executed by backward inferences. Limitation of this method obviously lies on the performance of inference mechanism, other problem maybe occurs in adding, removing another potential errors that will demand a considerable modification on the rules base. For our development, as suggested in [3], we intent to separate the analysis method (cf. Section 2.3 and 2.4) and the representation of errors modes using a group of four data files in format XML detailed below:

Questionnaire.xml : proposing to represent a list of questions from which we could evaluate the Common Performance Conditions (see Section 2.2 in following).

Phenotype.xml : proposing to maintain the phenotypes and its antecedents (cf. Figure 2).

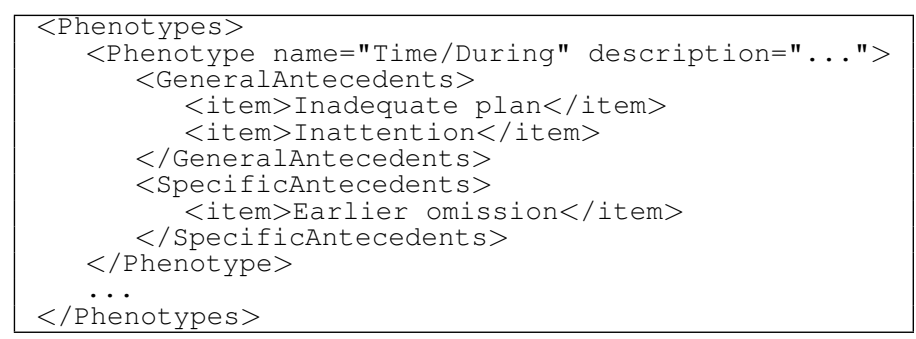

Figure 2: Representation of phenotypes

Genotype.xml : containing all possible causes classified in three groups (M,T,O), each group is then detailed into several categories. The important point is that this data file also represents relations between each consequent and its antecedents (cf. Figure 3).

Repartition.xml : proposing to determine repartition of specific antecedents (cf. Figure 4) in three factors $(\mathrm{M}, \mathrm{T}, \mathrm{O})$ which serves to initialize the mass of each specific antecedent as a probable cause (cf. Section 2.4).

Finally, in considering that CREAM is naturally a flexible method and adaptable to different analysis contexts, this strategy of classification scheme representation permits customize the scheme without any modification on analysis method. 


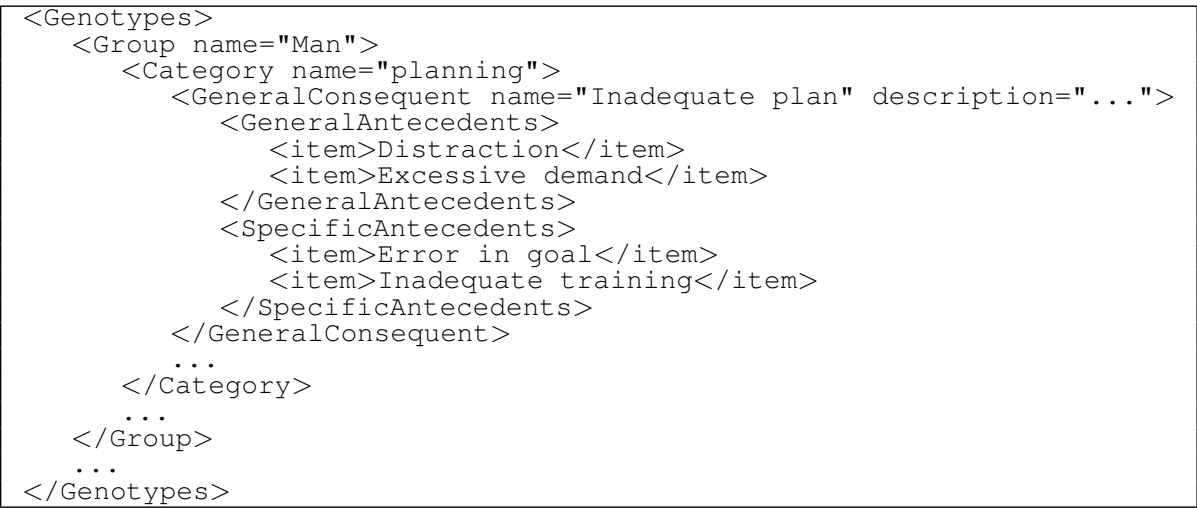

Figure 3: Representation of genotypes

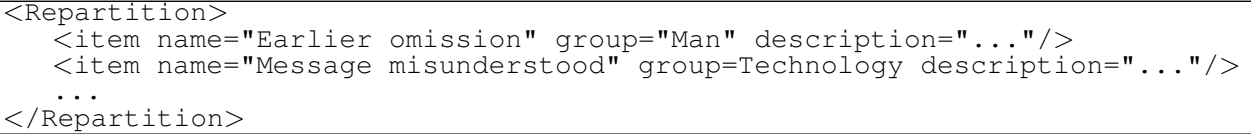

Figure 4: Repartition of specific antecedents in three factors $(\mathrm{M}, \mathrm{T}, \mathrm{O})$

\subsection{Define the Common Performance Conditions (CPC's)}

In CREAM, Hollnagel highlighted that the context strongly influence human actions. It is therefore essential to take into account the description of virtual environment in which the human learner is immersed. The objective is to determine how each factor $(\mathrm{M}, \mathrm{T}, \mathrm{O})$ influences the training context. Here, we are inspired from the proposition presented in [5] using a predefined questionnaire which will be answered by the teacher before training session (cf. Figure 5). Next, each factor will be assigned one

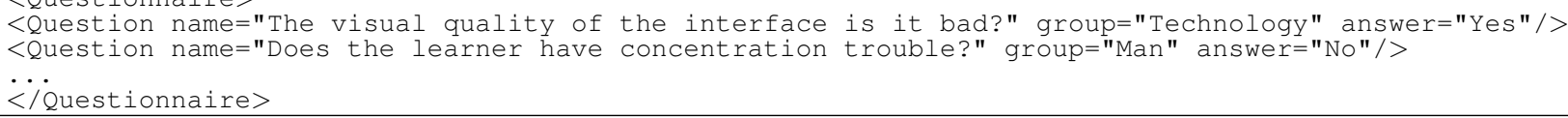

Figure 5: Define the CPC's by questionnaire [5]

coefficient calculated using formula below:

$$
\text { Coefficient }_{\text {group } i}=\frac{\text { Number of Yes answers associated to group } i}{\text { Total number of Yesanswers }}
$$

where group $i$ is respectively in (Man, Technology, Organization). These values permit define the most probable factor leading to erroneous actions.

\subsection{Modelling of Consequent-Antecedent Relations}

One advantage of CREAM lies on its recursive analysis approach, rather than strictly sequential in compare with other traditional analysis methods. So that, it also conducts to a non-hierarchical data structure to connect the direct as well as indirect links: (i) between a phenotype and its antecedent; and (ii) between a consequent and its antecedents. Figure 6 shows our model to represent the connection between consequent-antecedent.

Here, we are going to construct a causal graph where we use the term node to point to either a consequent or an antecedent. Each node is described by its name; the group of errors modes that it is associated and its category in group; the description in text helps better explain the error's semantics in particular context. The boolean attribute terminal permit to identify if that is a terminal-cause or not. The most important is that, each node contains two lists: one includes its antecedents, other points to its consequents, in others words, they represent edges in/out one node in the causal graph. At last, each node must also 


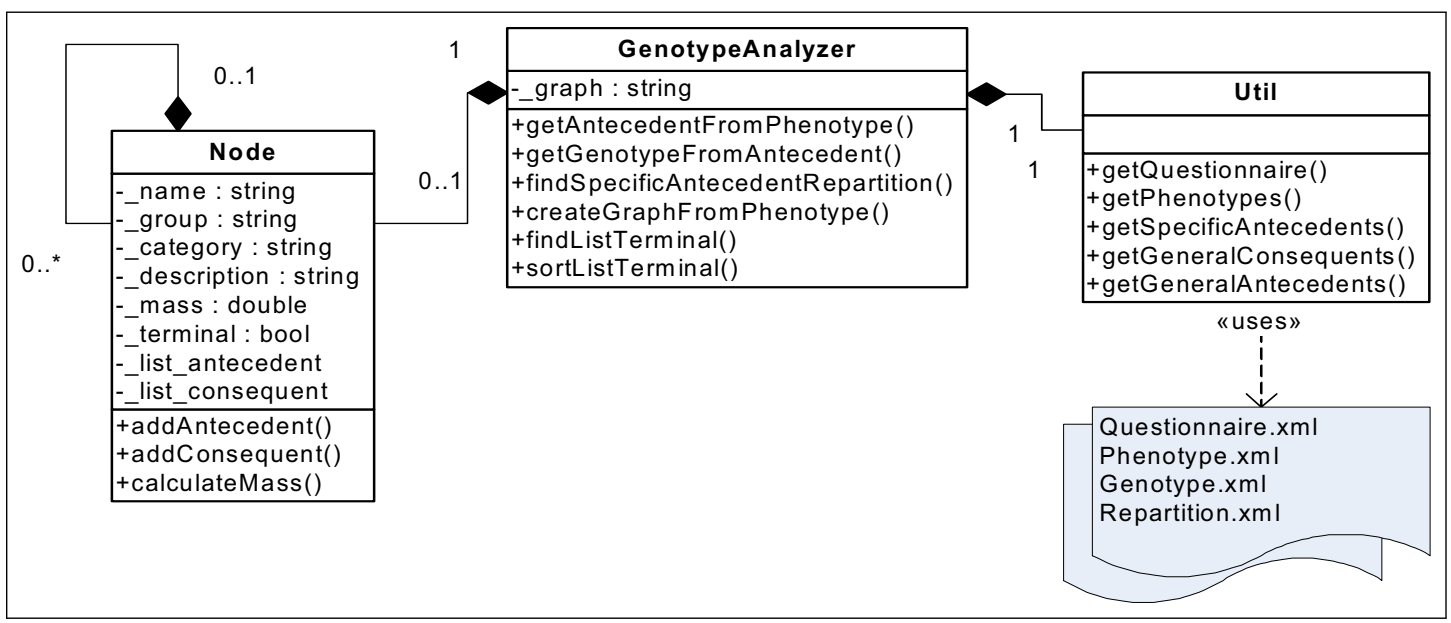

Figure 6: UML diagram for modeling consequent-antecedent links

include a value of mass which represent the certitude of choosing this node as a probable cause. The two methods addAntecedent() and addConsequent() serve for maintaining the two lists of antecedents and consequents of one node. Note that once a node calls the method addAntecedent() serving for adding a "parent" node like one of its antecedents, this node will also add itself to the consequents list of the "parent" node (using the method addConsequent() of the parent node), the value of the attribute terminal then will be set to false.

\subsection{Search for the Causes}

The retrospective analysis is executed by a GenotypeAnalyzer containing graph attribute which is initialized by pointing to the phenotype input (root node), then the analyzer calls accurate methods to find the root causes (the nodes with the attribute terminal having value false). This mechanism is presented below (cf. Algorithm 1).

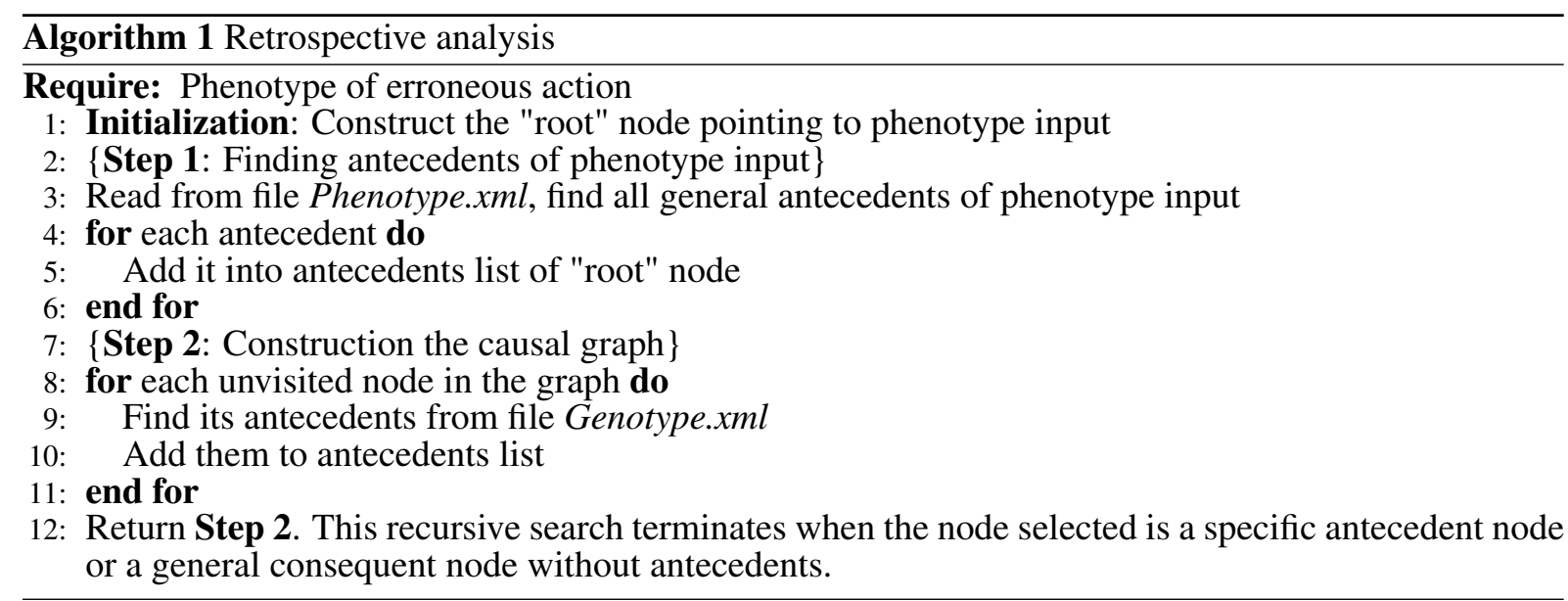

With this algorithm, we finally attain a causal network where each node is associated with its antecedents and consequents. The "leaves" are terminal nodes (or "root" causes) whose antecedents list is empty. In order to calculate the certitude of choosing each node as a probable cause, we inherit the proposition presented in [5] using Dempster-Shafer's evidence theory:

$$
\operatorname{mass}(a)=\text { coefficient }(g(a)) * \sum_{\forall c \in \operatorname{Cons}(a)}\left(\frac{\operatorname{mass}(c)}{\sum_{\forall i \in\{M, T, O\}}\left(\operatorname{coefficient}(i) * n_{i c}\right)}\right)
$$

where: 
- $\operatorname{mass}(a)$ : mass of antecedent $a$

- $g(a)$ : group of $a$

- $\operatorname{Cons}(a)$ : consequents list of $a$

- coefficient $(i)$ : coefficient of group $i$ calculated in Formula 1

- $n_{i c}$ : number of antecedents of $c$ classified in group $i$

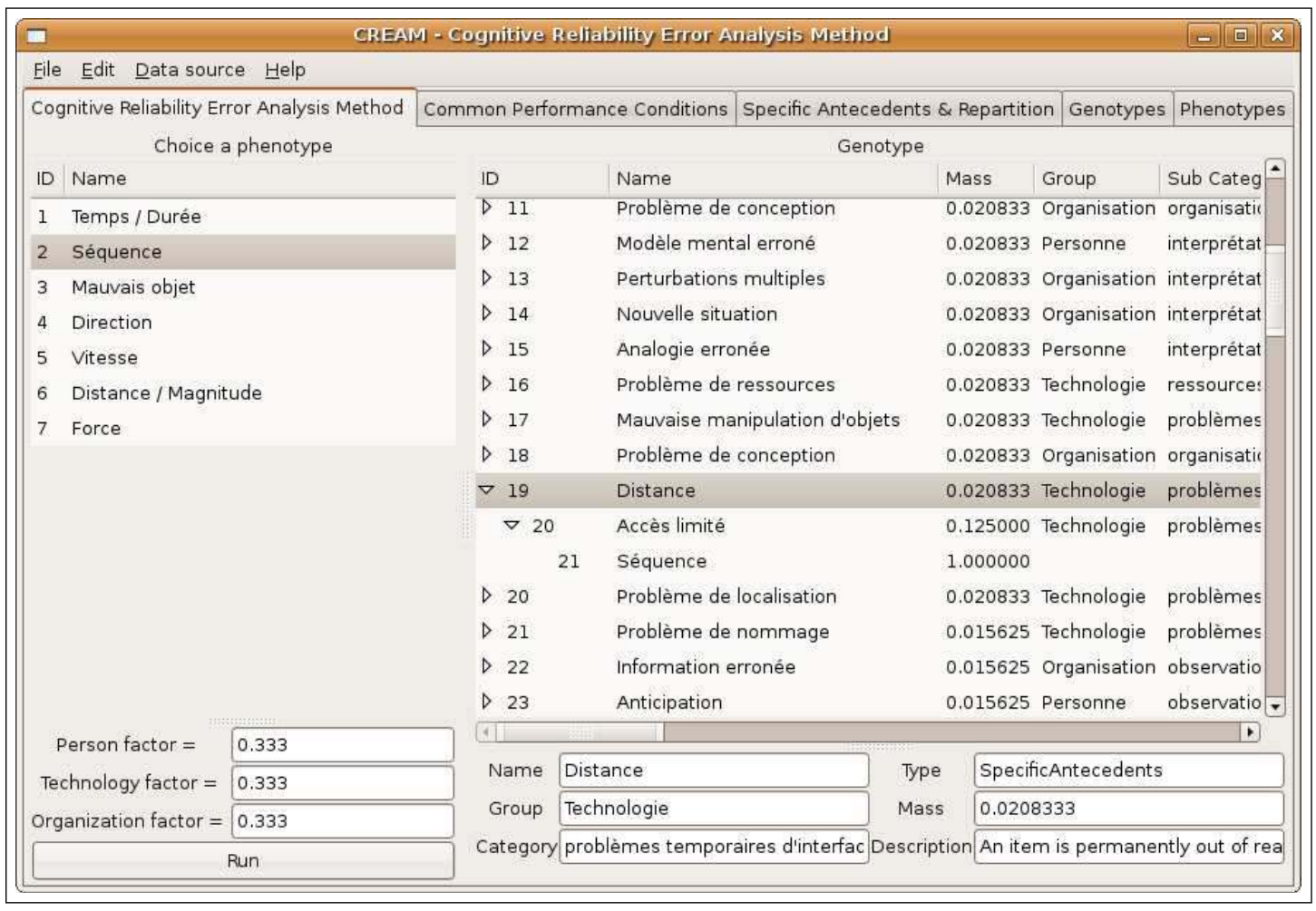

Figure 7: CREAM Explorer

Finally, the Figure 7 illustrates our tool - CREAM Explorer which was developped in this phase permitting to maintain the errors scheme, answer the questionnaire for define the CPC's and execute the retrospective analysis.

\section{Integration of Retrospective Analysis into our existing ITS}

\subsection{Learner's Plans Recognition}

In order to detect the erroneous actions realized by a human learner, it is indispensable to know:

(i) the learner's activities in the past;

(ii) his current action (in the meaning that the action has just been done);

(iii) the actions that the human learner intents to do in according to a predefined correct procedure.

Our existing ITS as proposed in [2] bases on the model MASCARET [1] where we used an multi-agent system to simulate collaboration between human learners and agents during their realization of tasks. Learners are gathered in team consisting of several predefined roles, every role contains a number of tasks associated eventually with accurate resources, every leaner also owns an epistemic memory containing all actions realized in the past, etc. Finally, we could retrieve from MASCARET following informations relating to learner's plan in VET: 
- action(s) before: learner's action(s) in the past (note that, in MASCARET, every action is eventually associated with its accurate resource(s))

- current action: action has just been done by learner

- action(s) correct (according to role): action(s) must be done by learner in his role(s)

- action(s) correct (according to plan): action(s) may be done by learners in the context. Here, it is essential to make distinction betweens action(s) correct according to role and action(s) correct according to plan. In the first case, because the learner could play several roles, it represents all correct actions that the system expects from the learners. The second one concerns the cases where there are more than one learner in VET to realize together a mission. Therefore, in this case, it is possible that a leaner performs a correct action according to the plan but it is not correct in compare to his role.

- next correct action(s) in the role: next action(s) must be done by learner in his role(s)

- full correct plan: description of all accurate actions (associated with resources) in predetermined procedure that the learner must respect.

In next section, we present our mechanism for mapping erroneous actions detected by our existing ITS with Hollnagel's classification scheme of errors modes.

\subsection{Classification of Erroneous Actions according to the Scheme of CREAM}

\section{Erroneous Actions in Phenotype "Sequence"}

According to Hollnagel, performing an action at the wrong place in a sequence or procedure is a common erroneous action, and it is more realistic in our context of simulation of procedural and collaborative work. The "Sequence" problem consists of several specific effects: Omission (an action was not carried out); Jump forward/ Jump backwards (actions in a sequence were skipped/carried out again); Repetition (the previous action is repeated); Reversal (the order of two neighbouring action is reversed); Wrong action (an extraneous or irrelevant action is carried out). We present in following our mechanism to detect erroneous actions in phenotype "Sequence":

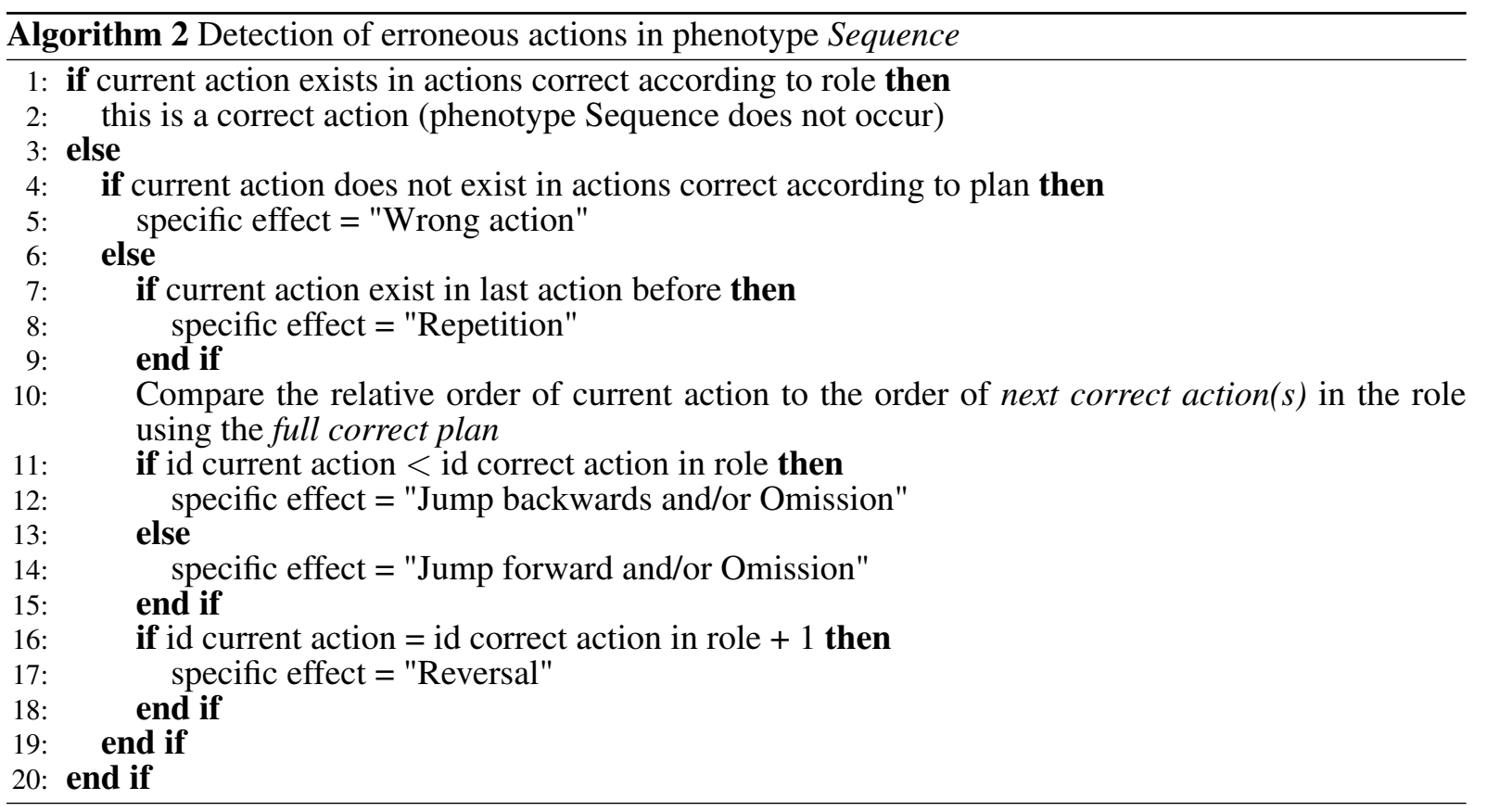

Erroneous Actions in Phenotype "Wrong object"

In [3], the author clarified that "action at wrong object" is one of the more frequent error modes, such as pressing the wrong button, looking at the wrong indicator, etc. In our context, during realisation of 
collaborative work, it is possible that learner performs a correct action but on a wrong object. Therefore, the detection of erroneous actions in phenotype "Wrong object" must be implemented independently with the detection of phenotype "Sequence". This phenotype is detailed into following specific effects: Neighbour/Similar object (an object that is proximity/similar to the object that should have been used); Unrelated object (an object that was used by mistake).

In order to detect erroneous actions in phenotype "Wrong object", we use the same principle presented in the case of phenotype "Sequence" by using following informations retrieved from model MASCARET:

- current resource: resource associated with current action

- resource(s) correct (according to role): resource(s) must be used by learner in his role(s)

- resource(s) correct (according to plan): list of resource(s) associated with all action(s) in action(s) correct according to plan.

Our algorithm is detailed in following:

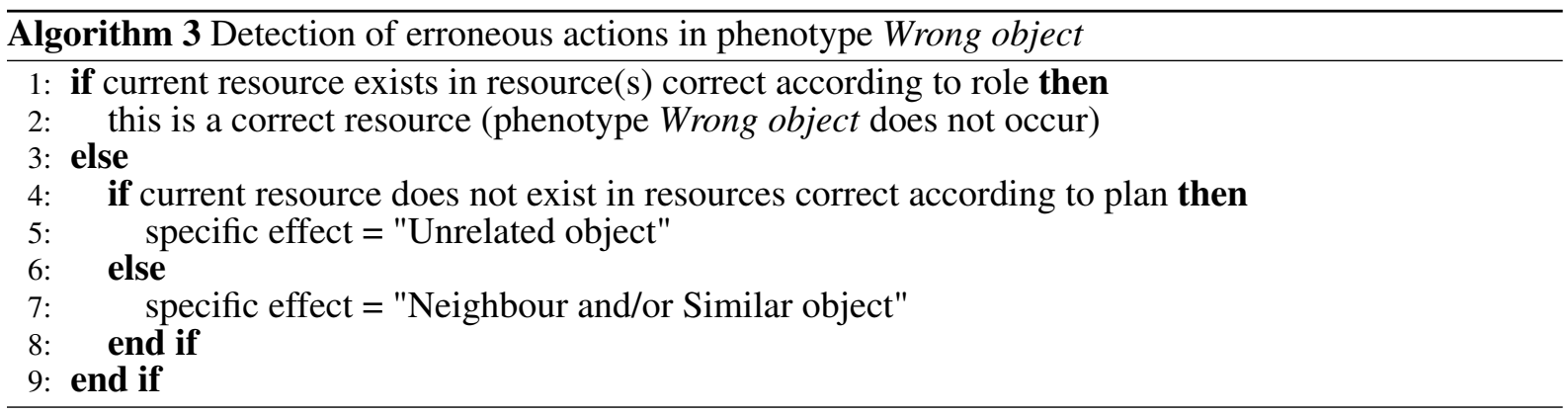

\section{Erroneous Actions in Phenotype "Time/During"}

The phenotype Time/During is divided in several specific effects: Too early/ Too late (an action started too early/too late); Omission (an action that was not done at all); Too long/Too short (an action that continued/was stopped beyond the point when it should have been). Hollnagel noted that the error modes of timing and duration refer to a single action, rather than to the temporal relation between two or more actions. In our context, the realization of tasks in model MASCARET is sequential, therefore, an action is considered to be too early when it was realized before several actions in plan; also, action(s) are considered to be omitted when they were not carried out.

Finally, in order to detect erroneous actions in phenotype Time/Durring, we propose that:

- action having specific effect Jump forward also has specific effect Too early

- action described by specific effect Omission (in error mode Sequence) will be considered as an action having specific effect Omission (in error mode Time/During)

\subsection{Experiment \& Results}

In order to evaluate our integration of retrospective analysis into ITS, we take place in GASPAR application [6] whose objective aims at simulate aviation activities by virtual reality. The learners are immersed in virtual environment simulating the aircraft carrier in order to realize together the tasks. During the realization of these collaborative works, our ITS follows the learners and then apply the algorithms depicted above for detecting learner's erroneous actions. Next, for interpreting the causes of errors, we use the classification scheme of error modes proposed in [5] which were particularly adapted to VET. Table 1 and Table 2 respectively illustrate results of retrospective analysis for the phenotype Sequence and Wrong object.

We change coefficients of three factors (M,T,O) for evaluating how CPC's influence the analysis result. For each phase in analysis process, we select and display the most probable cause by ordering mass values. 


\begin{tabular}{|c|c|}
\hline Coefficient (M,T,O) & Causal links \\
\hline \multirow[t]{3}{*}{$(0.333-0.333-0.333)$} & $\begin{array}{l}\text { 1, Design failure }(0.125) \rightarrow \text { Inadequate scenario }(0.125) \rightarrow \text { Se- } \\
\text { quence }\end{array}$ \\
\hline & $\begin{array}{l}\text { 2, Adverse ambient condition }(0.125) \rightarrow \text { Inattention }(0.125) \rightarrow \\
\text { Sequence }\end{array}$ \\
\hline & $\begin{array}{l}\text { 3, Long time since learning }(0.042) \rightarrow \text { Memory failure }(0.125) \rightarrow \\
\text { Sequence }\end{array}$ \\
\hline \multirow[t]{3}{*}{$(1-0-0)$} & 1 , Other priority $(0.2) \rightarrow$ Memory failure $(0.2) \rightarrow$ Sequence \\
\hline & $\underset{\rightarrow \text { Sequence }}{2, \text { Error in mental model }(0.067) \rightarrow \text { Faulty diagnosis }(0.2)}$ \\
\hline & $\begin{array}{l}3 \text {, Erroneous analogy }(0.067) \rightarrow \text { Faulty diagnosis }(0.2) \rightarrow \text { Se- } \\
\text { quence }\end{array}$ \\
\hline \multirow[t]{3}{*}{$(0-1-0)$} & 1 , Equipment failure $(0.1) \rightarrow$ Access problems $(0.5) \rightarrow$ Sequence \\
\hline & 2 , Distance $(0.1) \rightarrow$ Access problems $(0.5) \rightarrow$ Sequence \\
\hline & $\begin{array}{l}3 \text {, Localisation problem }(0.1) \rightarrow \text { Access problems }(0.5) \rightarrow \text { Se- } \\
\text { quence }\end{array}$ \\
\hline$(0-0-1)$ & 1 , Noise $(1) \rightarrow$ Communication failure $(1) \rightarrow$ Sequence \\
\hline
\end{tabular}

Table 1: Causal links of phenotype Sequence

\begin{tabular}{|c|c|}
\hline Coefficient (M,T,O) & Causal links \\
\hline \multirow[t]{3}{*}{$(0.333-0.333-0.333)$} & 1, Access problems $(0.125) \rightarrow$ Wrong object \\
\hline & $\begin{array}{l}2, \text { Design failure }(0.125) \rightarrow \text { Inadequate scenario }(0.125) \rightarrow \\
\text { Wrong object }\end{array}$ \\
\hline & $\begin{array}{l}\text { 3, Adverse ambient condition }(0.042) \rightarrow \text { Inattention }(0.125) \rightarrow \\
\text { Wrong object }\end{array}$ \\
\hline \multirow[t]{3}{*}{$(1-0-0)$} & 1 , Fatigue $(0.1) \rightarrow$ Performance variability $(0.2) \rightarrow$ Wrong object \\
\hline & $\begin{array}{l}\text { 2, Virtual reality sickness }(0.1) \rightarrow \text { Performance variability }(0.2) \\
\rightarrow \text { Wrong object }\end{array}$ \\
\hline & $\begin{array}{l}\text { 3, Anticipation }(0.05) \rightarrow \text { Wrong identification }(0.2) \rightarrow \text { Wrong } \\
\text { object }\end{array}$ \\
\hline$(0-1-0)$ & 1, Access problems $(0.5) \rightarrow$ Wrong object \\
\hline$(0-0-1)$ & 1, Noise $(1) \rightarrow$ Communication failure $(1) \rightarrow$ Wrong object \\
\hline
\end{tabular}

Table 2: Causal links of phenotype Wrong object

\section{Conclusion \& Future Work}

In this paper, we proposed an approach to modelling the Cognitive Reliability and Error Analysis Method (CREAM). We separated the representation of classification scheme of erroneous actions and the analysis method; therefore, our description of errors modes is adaptable to different training context without any modification on analysis method. We started by defining the Common Performance Conditions, then the direct and indirect relations between consequent-antecedent are modelled using a non-hierarchical data structure. Finally, the most probable cause-effect links could be found using Dempster-Shafer's theory presented in [5].

In order to integrate the retrospective analysis described above into our existing ITS, we based on the model MASCARET to retrieve information concerning learner's plans and then detect erroneous actions. Finally, we presented our proposition to mapping erroneous actions with Hollnage's classification. The experimental results in GASPAR project are also presented. So that, in addition to the detection and tagging of erroneous actions, the ITS could furthermore indicate the path of probable cause-effect 
explaining reasons that the errors occur.

In the future work, we will concentrate our attention on evaluation of MASCARET so that this model could permit to describe more complex tasks in taking into account other factors such as force, distance, speed, direction, etc. Hence, other different types of errors modes could be detected and then explained using the retrospective analysis.

\section{Acknowledgement}

This article is an extended version of our paper [8] published in Proceedings of the $3^{\text {rd }}$ International Conference on Virtual Learning (ICVL'08). The authors would like to thank the Scientific Committee of ICVL'08 (Chaired by Dr. Grigore Albeanu) that recommended the publishing of our extended work in IJCCC.

\section{Bibliography}

[1] R. Querrec, C. Buche, E. Maffre, and P. Chevaillier, Multiagents systems for virtual environment for training: application to fire-fighting, International Journal of Computers and Applications (IJCA), pp. 25-34, June 2004.

[2] C. Buche and R. Querrec, Intelligent tutoring system for MASCARET, Simon Richir and Bernard Taravel, editors, 7th Virtual Reality International Conference (VRIC'05), pp. 105-108, April 2005, Laval, France.

[3] E. Hollnagel, Cognitive Reliability and Error Analysis Method, Oxford: Elsevier Science Ltd, 1998.

[4] N. El-Kechaï and C. Després, A Plan Recognition Process, Based on a Task Model, for Detecting Learner's Erroneous Actions, Intelligent Tutoring Systems ITS 2006, pp. 329-338, June 2006, Jhongli, TaĂŻwan.

[5] N. El-Kechaï and C. Després, Proposing the underlying causes that lead to the trainee's erroneous actions to the trainer, EC-TEL : European Conference on Technology Enhanced Learning, pp. 41-55, September 2007, Crète, Grèce.

[6] N. Marion, C. Septseault, A. Boudinot and R. Querrec, GASPAR : Aviation management on an aircraft carrier using virtual reality, Cyberworlds, 2007.

[7] R.D. Serwy and E.M. Rantanen, CREAM Navigator, http://www.ews.uiuc.edu/serwy/cream/v0.6beta/, version 0.6, September, 2007.

[8] T.H. Trinh, C. Buche and J. Tisseau, Modeling of errors realized by a human learner in virtual environment for training, $3^{\text {rd }}$ International Conference on Virtual Learning ICVL 2008, pp. 71-80, Octobre 31, Constanta, Romania.

Thanh-Hai Trinh, received his MSc in Artificial Intelligent \& Multimedia at the Francophone Institute for Computer Science. He is currently a PhD student in CERV (Virtual Reality European Centre held at Brest, France). His current research interests are in the applications of multi-agent systems and artificial intelligent in virtual environements for training.

Cédric Buche is a Professor Assistant in Computer Science and works at the CERV. He works on the use of the behavior modeling agent applied to virtual environment for human learning. He is the leader of the ITS project in MASCARET.

Ronan Querrec is Professor Assistant in Computer Science and works at the CERV. His reasearch work is about virtual environment for training. In this theme, he works on the MASCARET project, a virtual environment meta-model.

Jacques Tisseau is Professor in Computer Science at the Engineer School of Brest (ENIB) where he leads the Computer Science for Complex Systems Laboratory (LISyC). His research focus on autonomous virtual entities, interaction with these entities and epistemology of virtual reality. 